\title{
Explaining the impact of conductor surface type on wet weather HVDC corona characteristics
}

\section{Journal Article}

Author(s):

Schultz, Tim (1); Pfeiffer, Martin D.; Hedtke, Sören (1); Franck, Christian (i)

Publication date:

2016-02

Permanent link:

https://doi.org/10.3929/ethz-b-000185185

Rights / license:

Creative Commons Attribution-NonCommercial-NoDerivatives 4.0 International

Originally published in:

Journal of Electrostatics 79, https://doi.org/10.1016/j.elstat.2015.12.003 


\title{
Optical Investigation Methods for Determining the Impact of Rain Drops on HVDC Corona
}

\author{
Tim Schultz ${ }^{\mathrm{a}}$, Martin Pfeiffer ${ }^{\mathrm{a}}$, Christian M. Franck ${ }^{\mathrm{a}}$ \\ ${ }^{a}$ Power Systems and High Voltage Laboratories, ETH Zurich, Physikstrasse 3, 8092 Zurich, Switzerland
}

\begin{abstract}
Corona behavior of HVDC overhead lines plays a significant role when dimensioning transmission lines. Rain constitutes an important study case since corona effects (e.g. losses, discharge amplitudes) are considerably affected. In this paper, imaging methods are introduced to investigate the impact of rain on corona behavior. Geometrical properties of rain drops on a stranded conductor were extracted. UV-images were used to precisely locate discharges. The methods' capabilities are demonstrated using data from an indoor test line during a simulated rain shower. Optical and electrical data were correlated. These methods aim to support the development of surfaces with favorable corona properties.
\end{abstract}

Keywords: HVDC, corona, ion current, rain drops, image processing

\begin{tabular}{ll} 
& List of Abbreviations \\
\hline AN & Audible Noise \\
CL & Corona Losses \\
DSLR & Digital Single Lens Reflex Camera \\
PD & Partial Discharge \\
RI & Radio Interference \\
UV & Ultra Violet
\end{tabular}

\section{Introduction}

The promotion of renewable energy sources in Europe over the recent years lead to an increased demand for 35 5 transport capacity in the electricity grid [1]. To cope with this development, the construction of new HVDC lines as well as converting individual AC systems on multi-circuit lines to DC is planned [2]. As a consequence, corona behavior of HVDC lines is of great interest, since it con- 40 10 tributes to audible noise and radio interference as well as ion currents [3], which can reduce human sensation thresholds [4] and influence adjacent AC systems [5].

One of the main influencing factors of corona on overhead lines is rain [6]. Measurement data to study its effect 45 on the discharge behavior of overhead lines has been obtained through outdoor as well as indoor experiments.

Outdoor measurements were able to confirm a significant increase of ion current and electric field levels [7, 8, 9, 6, 10, 2] as well as a considerable influence on radio inter- 50 ference (RI) [11, 12] due to rain. However, the published data is limited and contains no detailed information that would allow to correlate rain drop properties and electrical corona characteristics.

\footnotetext{
*Corresponding author: Tim Schultz

Email address: tschultz@ethz.ch (Tim Schultz)
}

Indoor investigations in contrast, have mostly focused 25 on the fundamentals of electrical discharges from liquid points. In 1914 Zeleny investigated surface instabilities and electrical discharges on individual liquid points subjected to electric fields. He observed various discharge regimes and drop shapes that change as a function of the 30 applied electric field [13]. Further studies in this field were by Macky in 1931 [14] and English in 1948 [15. Macky concentrated on studying the deformation of free falling drops in electric fields. English supports Zeleny's analysis that with increasing electric fields, water drop points become unstable because electrostatic and gravitational forces exceed the surface tension force of the drop.

In 1964, Taylor published an extensive theoretical and analytical treatment of discharge phenomena of water drops in electric fields [16]. He established that liquid drops subjected to high field strengths approach a conical shape. Furthermore, he showed experimentally and analytically that the critical cone angle for the break-up of the surface of a water drop in an electric field is $98.6^{\circ}$.

In 1965 Akazaki investigated the impact of water drops for DC transmission line applications [17]. He used a smooth cylindrical aluminum conductor and primarily studied single water drops. He describes a transition from low repetition corona discharges to high repetition discharges as the voltage is increased. He also divides the noise produced from DC rain drop corona into "crackling" and "hissing" sounds, the occurrence of which differed depending on the applied voltage, the drop size and the location on the conductor. He also showed that after turning off the water supply, corona losses (CL) steadily decreased while RI increased. He attributed this to differences in the field distribution in the immediate vicinity of drops, which can lead to either high pulse repetition rates but low pulse 
amplitudes (pointy drops) or low repetition rates and high amplitudes (blunt drops).

Further valuable theoretical and experimental contributions to this topic were presented in [18, 19, 20, 21].115 A detailed discussion, however, would exceed the scope of this paper.

The conclusion of this literature survey is that the complexity of corona discharges from liquid drops has been recognized and studied in significant depths. Strong linksi20 between the geometric characteristics of water drops and the resulting corona phenomena have been established but detailed investigations were almost exclusively limited to individual drops on cylindrical, non-stranded conductors.

In this publication a collection of novel image gath-125 ering and processing methods that allow connections to be drawn between the shape, density, as well as distribution of rain drops on practical transmission line con-

75 ductors and their resulting electrical corona characteristics is presented. It intends to create a bridge between those references that looked at the fundamental characteristics of water drop corona at a rather microscopic level (e.g. [13, 14, 15, 16, 17]) and those that took a very macro80 scopic view, analyzing outdoor field test data on full-scale lines (e.g. [12, 11, 6, 2]).

The intended application of these methods is the selection and improvement of conductor surfaces with respect to their corona behavior during rain. It was shown in [22]

85 that reductions in DC CL of the order of 30 to $40 \%$ can be achieved with the use of special strand types and an increased surface roughness. It is clear that these differences are due to the impact of surface characteristics on the wetting and drop forming behavior. The underlying 90 reasons for these differences, however, are not yet well understood, which limits the systematic development of even more advantageous surface types.

In this paper, the results of a single set of measure- ${ }^{135}$ ments using a stranded conductor are presented to demon95 strate the capabilities of the new methods. The measurement was carried out indoors with a realistic rain simulation setup and includes the transition from dry conditions to a one hour rainy period and the subsequent drying-off of the conductor. Electrical quantities recorded are corona currents (as a measure of CL) and partial discharge pulse amplitudes (indicative of RI and audible noise (AN) [17]). Optical information includes an automated count of number, size, and cone angle of water drops, as well as it keeps track of discharges via UV images. Systematic investigations of other experimental parameters (e.g. rain intensity, voltage level, polarity) and conductor type are currently ongoing.

\section{Experiment Set-up and Procedure}

\subsection{Experimental Setup}

An experimental setup based on previous investigations $\$ 2$ 23. was used (Fig.11). It consists of an overhead line (1) that is attached to the laboratory walls by a rope system, which allows to adjust its height above ground continuously. The height of the line, $h$, is determined at the point of maximum sag in the center of the arrangement and was $1.095 \mathrm{~m}$ for this experiment. The sag was $5 \mathrm{~cm}$. Grounded fences with a height of $2.15 \mathrm{~m}$ were in parallel with the conductor (with a distance of $1.45 \mathrm{~m}$ to one side and $1.3 \mathrm{~m}$ to the other). The results presented in this paper were obtained using a single 264-AL1/34-ST1A conductor with a length of $6.8 \mathrm{~m}$ and a diameter of $22 \mathrm{~mm}$. Toroids (2) are used to suppress undesired corona activity at both line ends.

The line can be energized by a DC source (3), consisting of a switched AC power supply, a high voltage transformer and a two-stage Greinacher cascade.

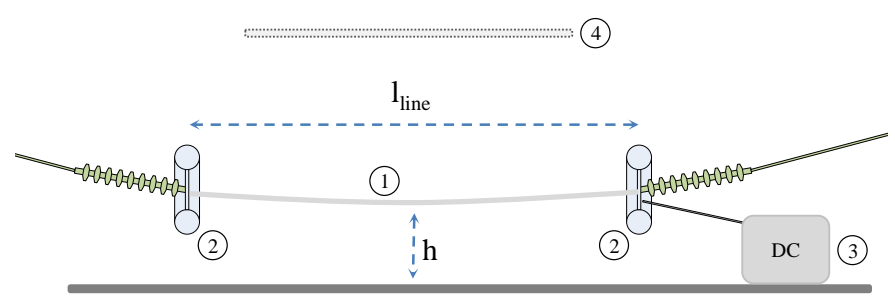

Figure 1: Schematic drawing of the experimental setup (side view not to scale).

The voltage is measured using a resistive divider which is connected to the measurement computer with an analog digital converter. Additionally, the total corona current is recorded using an optically decoupled current probe by the ETH spin-off Kametech that measures the current flow between DC source and energized line. The current measurement uses a low pass filter (cut-off frequency: $1 \mathrm{~Hz}$ ). The partial discharge (PD) amplitude is measured with an Omicron MPD600 system calibrated and used in accordance with IEC 60270.

Rain is simulated using a rain dispenser (4), constructed by Straumann for his work on AC conductor noise emission 24. It consists of a drop dispenser mounted at the $10 \mathrm{~m}$ high ceiling, generating drops at roughly 500 locations on an area of about $0.6 \mathrm{~m} \mathrm{x} 4 \mathrm{~m}$, and a grounded diffusor grid, mounted $4 \mathrm{~m}$ above ground. The diffuser grid reduces the mean drop size and increases the drop size distribution, bringing it closer to the characteristics of natural rainfall [25]. The water is pumped to the drop dispenser from a tank and collected in a pool below the line. The rain intensity can be adjusted with a valve. The total water flow to the dispenser is monitored with a digital flow meter. For the presented results, a fixed rain intensity of approximately $12 \mathrm{~mm} / \mathrm{h}$ was used (based on ground level measurements at 5 positions along the line). Additionally, a movable rain blocker was constructed that can be remotely inserted below the drop dispenser and divert the water away from the overhead line, thereby allowing the rain to be switched completely "on" and "off" within approximately 5 sec. 


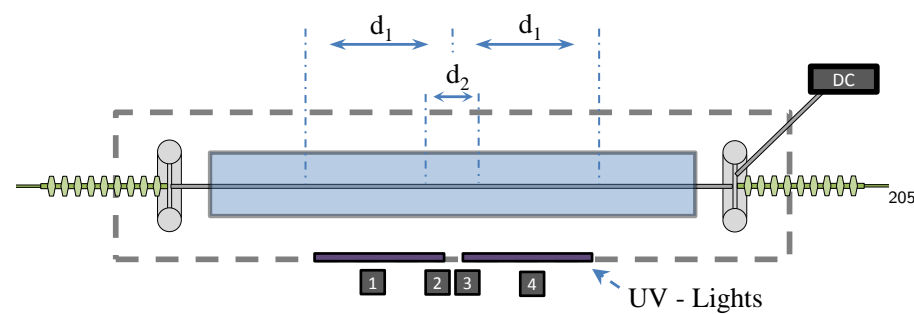

Figure 2: Schematic drawing of the experimental setup (top view not to scale).

For the optical part of the setup, four different cameras are installed, whose positions are indicated in Fig.2 Two digital single lens reflex cameras (DSLRs) $(1,4)$ are $_{215}$ placed $0.375 \mathrm{~m}$ to the right and the left of the center of the conductor (mounted at the same height as the conductor). The zoom of each camera was adjusted such that a length of $0.75 \mathrm{~m}$ of the overhead line was observed (labeled $d_{1}$ ). Both cameras can be triggered by the measuring ${ }_{220}$ computer system. Two additional digital compact cameras were placed in the center of the arrangement and focused on a $0.2 \mathrm{~m}$ section of the line (labeled $d_{2}$ ), one of which is used to record through a corona scope (Forsyth Electro). This is necessary since the scope is not capable of recordlight in the UV range which is typically emitted by corona discharges while it reduces the light of other frequencies. The result is then converted into visible light. The scope is sensitive enough to visualize discharges of less than $20 \mathrm{pC}$ from $5 \mathrm{~m}$ distance 26 . Sample pictures are shown in Section 3.1

To enhance visibility of the rain drops, a fluorescent contrast agent (fluorescein, concentration $70 \mathrm{mg} / \mathrm{l}$ ) was added to the tap water that was used for the artificial rain. In addition, two UV LED bars, placed on the ground at the safety fence, illuminated the conductor. This position was chosen to minimize reflections and improve the contrast of pendant drops. To ensure that the DC corona behavior is not changed by the contrast agent, tests using distilled ducted. Repeated measurements with the three different liquids showed that differences in the measured corona currents for various voltage levels were in the same order of ${ }^{240}$ magnitude as the statistical spread of measurements conducted with one liquid type alone (less than $10 \%$ for a voltage of around $135 \mathrm{kV}$ ).

\subsection{Measurement Procedure}

Prior to any measurement, the conductor was cleaned with lint-free wipes and ethanol and its height was checked. The start of the rain system was prepared by moving the rain blocker below the drop dispenser as well as preparing the fluorescein solution in the tank.

In the next step, the voltage was ramped up to the test level of $(137 \mathrm{kV}$, corresponding to a maximum conduc- cylinder of equivalent outer diameter) and the total corona current (for the dry conductor) was checked. In case the dry corona current exceeded $0.2 \mu \mathrm{A} / \mathrm{m}$, the cleaning procedure was repeated and the corona current checked again.

Afterwards, the pump was started and the desired rain level was adjusted. To reach a constant rain rate before exposing the overhead conductor to rain, the pump was running for 10 minutes with the removable rain blocker in place. Data recordings of these 10 minutes provide the dry corona behavior of the conductor as a reference. From this point on, the overhead line was exposed to rain for $1 \mathrm{~h}$. The start of the rain is indicated in all figures as $t=0 \mathrm{~min}$.

To initiate the drying phase, the rain blocker was inserted again and the pumps were switched off. In this way, a quasi-instantaneous rain stop was reached.

The condition that marks the end of the drying process and hence of the measurement was again the threshold of $0.2 \mu \mathrm{A} / \mathrm{m}$.

During the whole measurement procedure, line voltage, ion current, PD as well as water flow rate to the rain dispenser were measured. Additionally, photos were captured at a rate of 1 per second by the DSLR cameras and videos recorded at 25 frames per second.

\section{Optical Evaluation Methods}

During the conducted series of experiments, thousands of photographs and several hours of video material were recorded. The video material was subsequently parsed into individual frames. One of the key features of this paper is the introduction of algorithms to process a large number of images and return accurate information about the distribution and shape of the water drops retained on the conductor. The steps necessary to extract this information from the recorded footage are presented in this section. The accuracy of the algorithms has been manually validated by checking a large number of images from various phases of the experiment.

\subsection{Unprocessed Material}

A number of sample image sections from the DSLR cameras can be seen in Fig.3. The measurement was started with a dry conductor. Immediately after the rain started, small droplets on the upper side of the conductor were observed (1). With time, water accumulated and larger drops were formed, additionally a number of small droplets can be found on the lower side of the conductor (2). Further water supply leads to a movement of the larger drops to the lower edge of the conductor, where pendant drops are formed. Due to the high electric fields, Taylor cones appear. During the steady state phase (3), water is concentrated onto the lowest parts of the conductor. After the water supply is stopped, the drying process leads to a decrease in drop size and number (4). At the end of the drying process, only small traces of water between the strands of the conductor are visible. 

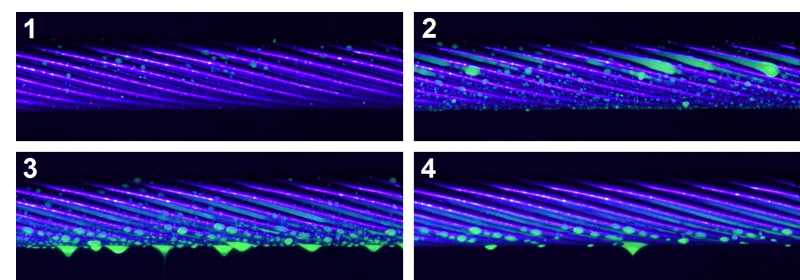

Figure 3: Unmodified excerpt of sample pictures from a DSLR cam-295 era: rain start $(1,2)$, steady state $(3)$ and drying process $(4)$.

A detail of a sample image taken through the UV scope 255 can be seen in Fig. 4. The discharges in this particular case start only from the lower edge of the conductor.

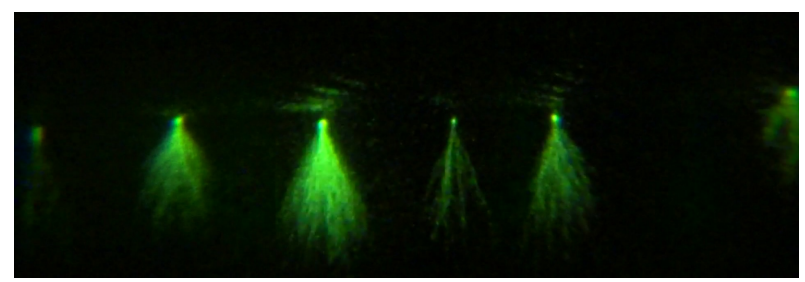

Figure 4: Unmodified excerpt of a sample picture from a compact camera through the UV scope (steady state rain).

\subsection{Image Processing}

All images, except those using the UV corona scope, were processed to detect drops on the surface (area "on") and lower edge (area "under") of the conductor. Further-315 more, projected 2D properties were determined including the projected area of drops, and in the case of drops in area "under", also a measure of their sharpness, as described further below.

Due to the dimensions of the conductor, only a small part of the each image is of interest. To reduce size $a_{320}$ therefore required processing time, for each measurement series the position of the conductor is identified and the images are cropped. In the next step, the lower and upper edge of the conductor are detected in each image and two areas are defined for separate evaluation: the pro- ${ }_{325}$ jected conductor surface (area "on") and an area below the lower conductor edge (area "under"). The latter area is of special interest since here the formation of large pen-

275 dant drops coincides with the highest conductor surface field strength. Both areas are then processed with a separate set of parameters to identify water drops and their geometric properties. For the area below the conductor, an additional detection of their sharpness is performed.

\subsubsection{Conductor Detection}

Two algorithms for conductor detection are implemented, a rough determination used to crop the raw images and a more sophisticated one to identify the borders of the areas "on" and "under".

For cropping, the uppermost and lowermost line that is covered by the conductor are determined by horizontal integration of the brightness values of the blue color channel of each image. A threshold can then be defined to determine an upper and lower limit. An additional margin above and below is used not to lose relevant information.

The conductor edges are then identified more precisely by a second algorithm. To respect the sag of the conductor, both conductor edges are determined for each pixel column, again by applying a threshold to the blue color channel. To smoothen the shape of the curves, a second order polynomial function is fitted to the lower edge pixel. To ensure that both edges are parallel, the upper edge is defined as the lower edge curve shifted by the mean number of pixel between both edges.

\subsubsection{Drop Detection}

After identifying the two areas, a drop detection algorithm is applied to both. Due to the contrast agent, rain drops have a distinctive color, which is why the algorithm distinguishes dry from wet conductor parts by analyzing 305 if pixels are part of a predefined part of a color space. In addition to the color, thresholds for brightness and size as well as contrast enhancement were used to improve the drop detection. The final boundary detection was aided by the object analysis functions available in the Matlab Image Processing Toolbox.

The projection of individual drops on the lower conductor edge partly overlapped such that multiple drops were occasionally recognized as one continuous drop. An additional algorithm was therefore implemented to split merged drops. This was done by checking the lower outline of each detected drop for significant peaks, indicating tips of individual drops.

\subsubsection{Drop Sharpness Determination}

Water drops deform in electric fields and approach a cone-like shape. To what extend they deform depends on the drop size as well as on the electric field strength. The deformation, on the other hand, influences the corona behavior at the drop, which in return influences the space charge production and thereby the electric field. The phenomena is thus fully coupled.

To measure the drop deformation, the lower edge of each drop was analyzed as illustrated in Fig.5. First, the tip of each drop is identified (indicated by (C)). After excluding the outermost parts of the drop (left of (A) and right of (E)) as well as a certain region to both sides of the peak (between (B) and (D)), two lines are fitted to the remaining outlines to the left and the right of the peak. The angle between both is defined as the drop angle $\alpha$. Drops with an angle smaller than $98.6^{\circ}$ are defined as Taylor cones according to [16] (see also Introduction).

\subsubsection{Demo Image}

Fig. 6] demonstrates the results of the drop detection and sharpness determination. It shows a length of approximately $0.1 \mathrm{~m}$ at $t \approx 40 \mathrm{~min}$ of the experiment presented 


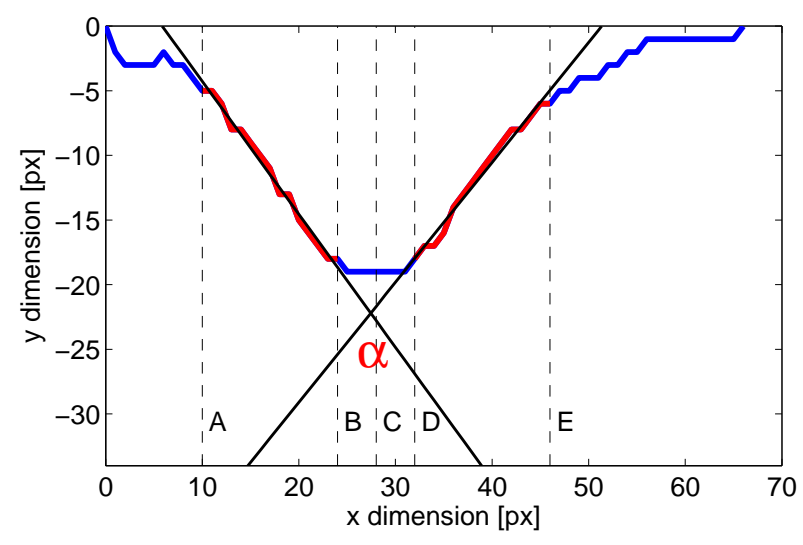

Figure 5: Illustration of method used to derive drop angle $\alpha$.

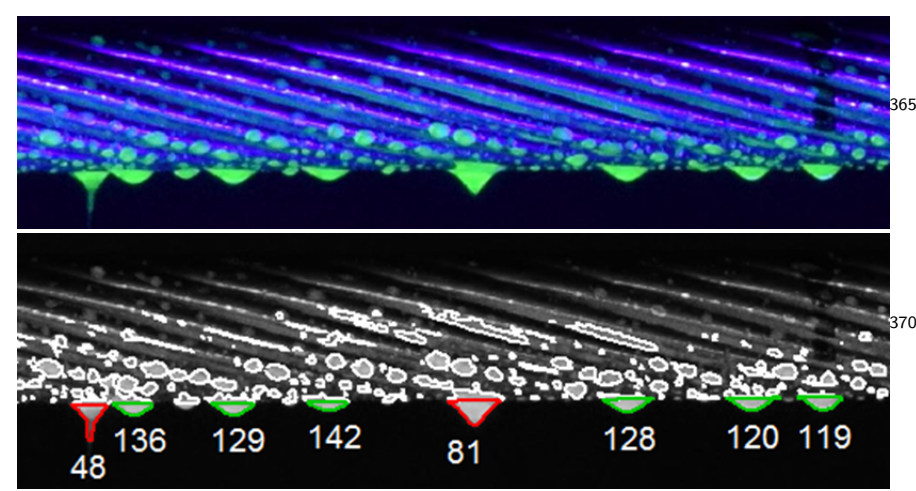

Figure 6: Sample image to demonstrate drop detection. Shown sec- ${ }^{375}$ tion is $\approx 0.1 \mathrm{~m}$. White: "on". Green: "under". Red: "under" with $\alpha<98.6^{\circ} 1$ st and 5th from the left). Numbers: drop angle $\alpha$.

in Section 2.2 Drops detected in the two regions are indicated by different colors. Furthermore, for all drops de- ${ }^{380}$ tected below the conductor, the drop angles $\alpha$ are shown in white numbers. Drops with an angle lower than $98.6^{\circ}$ are identified as Taylor cones. Not all drops detected in

345 the "under" region are plotted (a size threshold of $5 \mathrm{~mm}^{2}$ is used). A large number of images like this was generated and the accuracy of the presented detection algorithms successfully verified by manual evaluation.

\subsection{Image Processing (UV Scope)}

The camera equipped with a UV scope produced raw images like the one shown in Fig.4. Image processing methods were developed to identify the origin of discharges. Additionally, brightness and frequency of discharge processes may serve as an indicator for the corona intensity. However, a detailed evaluation of this data is not within the scope of this paper. Fig. 7 shows an overlay of the roots of detected discharges (marked by white crosses) over an image taken with a camera without the UV scope (showing the $0.2 \mathrm{~m}$ in the middle of the conductor, $d_{2}$ ). The ${ }_{400}$ upper figure shows the discharge locations integrated over the first 2 minutes immediately after rain start, while the lower figure shows the same result for two minutes starting from 50 minutes after rain start.

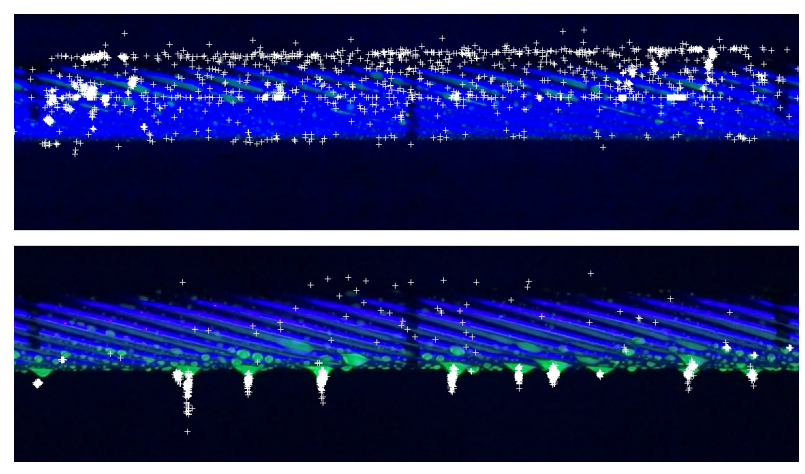

Figure 7: Discharges detected by evaluation of UV images (top: 0 min to $2 \mathrm{~min}$, bottom: $50 \mathrm{~min}$ to $52 \mathrm{~min}$ after rain start).

\section{Results}

\subsection{Overview of Entire Experiment Duration}

In this section, a selection of the electrical data and optical evaluation is presented for the entire experiment duration (from 10 minutes before the start of the rain until the very end of the drying process).

Fig. 8 illustrates the development of total corona current and apparent partial discharge (PD) pulse magnitudes. Optical data, namely number of drops and mean projected area for drops in area "on" and "under" is visualized in Fig.9 The rain phases starts at $t=0 \mathrm{~min}$, before which the corona activity is practically zero, apart from two short discharges. Even though a rare event during the conducted measurements, these were included since they illustrate the statistical character of dry HVDC corona with low ion currents but high PD levels.

Fig.10 depicts the distribution of drop angle $\alpha$ for the complete duration of the measurement. The minimum at $99.45^{\circ}$ is very close to the Taylor cone angle of $98.6^{\circ}$ and indicates that this critical value has a reduced occurrence likelihood compared to angles that are slightly lower or higher.

Fig. 11 shows the corona current as a function of the number of drops on and below the conductor respectively for the entire experiment duration (rain phase: 0 - $60 \mathrm{~min}$ ). This type of visualization combines data from Figs. 9 and 12 and can be used to investigate correlations between both measures as well as the influence of drops in different positions. It will be discussed further in Section 4.4 .

The experiment can be divided into 3 distinct phases: the starting phase, a (quasi) steady state and a drying phase. The start and end times of the phases are indicated by vertical red lines in Figs. 8 and 9 The following sections contain a short evaluation of these phases. The evaluation is not meant to be exhaustive, but its purpose is to highlight the capabilities of the presented optical methods.

\subsection{Rain Start}

At the beginning of the experiment $(t=0 \mathrm{~min})$, when the first drops hit the conductor, an immediate reaction 


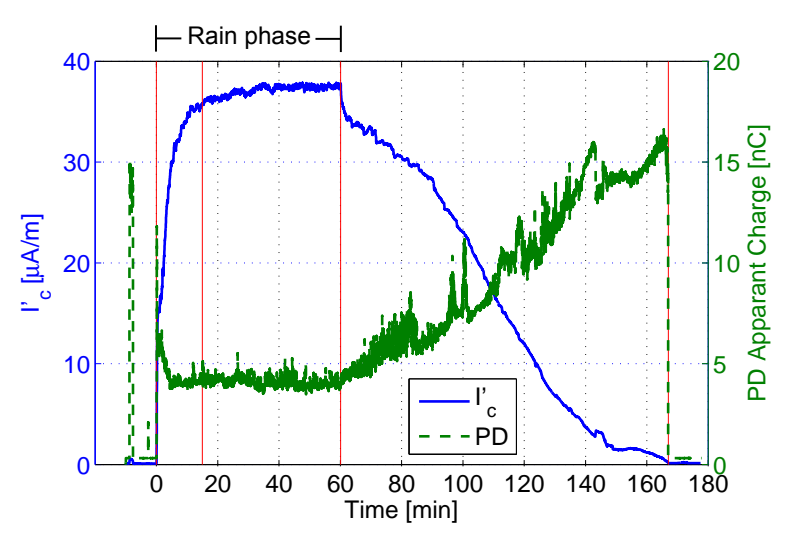

Figure 8: Corona current and partial discharge amplitude as a function of time for the entire experiment duration.
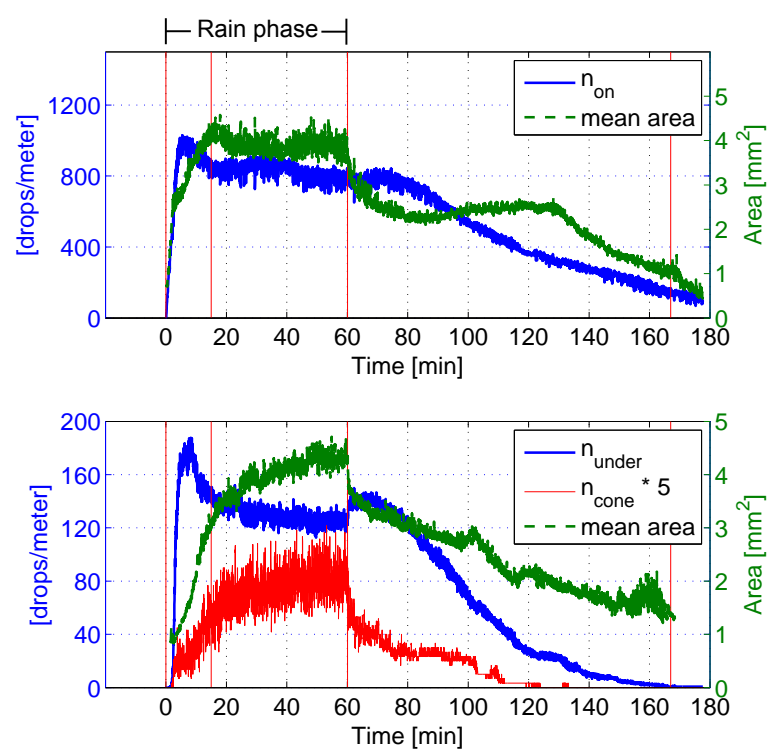

Figure 9: Number and mean projected area of drops as a function of time for the entire experiment duration (top: "on", bottom: "under").

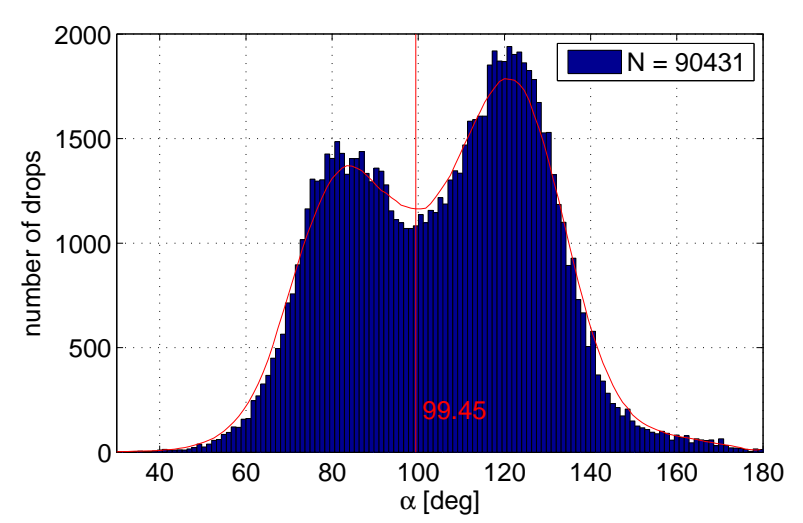

Figure 10: Distribution of $\alpha$ for entire experiment duration (neglecting drops with a projected area of less than $6.5 \mathrm{~mm}^{2}$ ). Based on analysis of 90431 drop images.
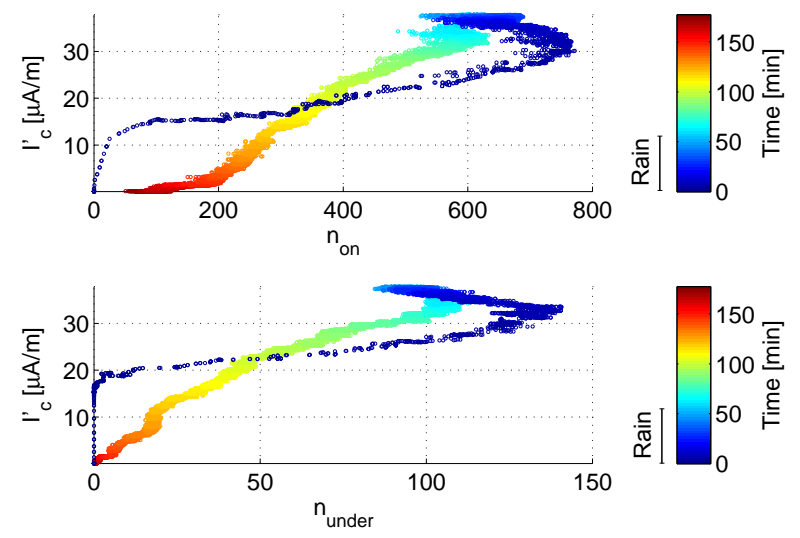

Figure 11: Correlation of corona current and number of drops (top: "on", bottom: "under") for entire experiment duration. Rain phase: 0-60 min (blue), drying phase: 60-180 min (green - red)

can be seen in the recorded electrical quantities (upper plot in Fig.12. The ion current shows a steep incline and within seconds exceeds $10 \mu \mathrm{A} / \mathrm{m}$. In parallel, the PD level jumps to more than $10 \mathrm{nC}$ only to settle down to about $6 \mathrm{nC}$ a moment later. After this immediate response, both measures reach a plateau-like phase, lasting for about a minute $(t \approx 1$ min to $t \approx 2 \mathrm{~min})$. Over the course of the next three minutes, PD levels decrease by about a third to a stable value of roughly $4.0 \mathrm{nC}$. During the same time the total ion current approximately doubles to $30 \mu \mathrm{A} / \mathrm{m}$, which is approximately $80 \%$ of its steady state value at 415 $t=60 \mathrm{~min}$.
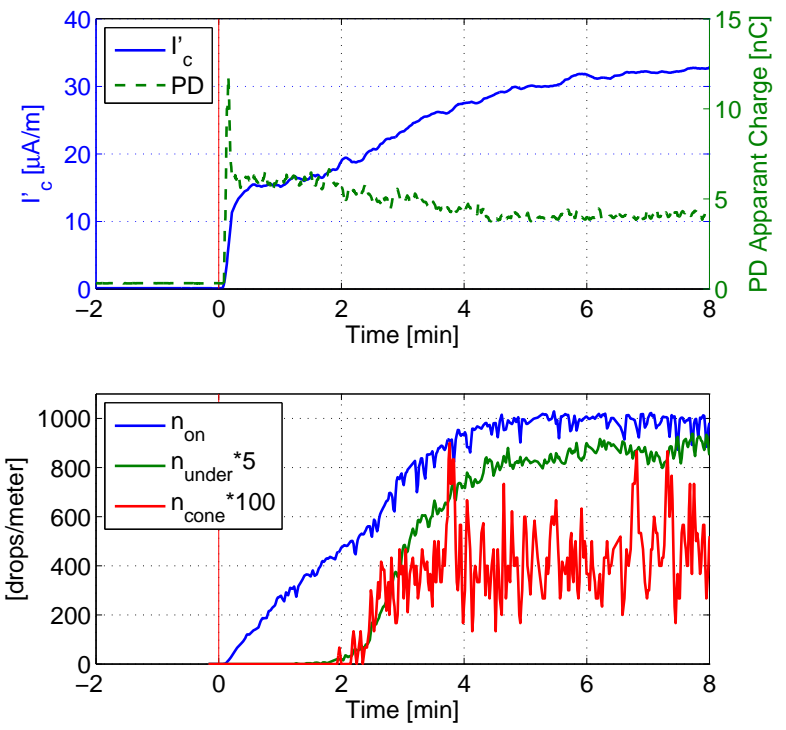

Figure 12: Start phase of the experiment. Top: Corona current and partial discharge amplitude as a function of time. Bottom: Number of drops and Taylor cones as a function of time

The optical quantities that were obtained using the method presented in this paper, show significant changes in this starting phase as well (cf. lower plot in Fig.12): The first drops on the conductor are identified almost ex- 
${ }_{420}$ actly at the moment when the electrical quantities start to change. From then on, the number of drops increases475 linearly and they grow in size. With a delay of about 1.5 minutes, the first drops form below the conductor and slightly later Taylor cones are detected. This coincides

${ }_{425}$ with the increase of the ion current and the decrease of PD after the plateau-like phase, described in the preced-480 ing paragraph.

The presented method makes it possible to link the characteristic development of electrical quantities to the

430 physical properties of the water drops. It indicates for example that small water droplets, like in the very beginning ${ }_{485}$ of the rain phase, cause considerable ion currents and PD.

The plateau-like behavior of CL and PD appears to be a transition phase, after which the corona behavior of 435 the line is largely influenced by the appearance of drops on the lower side of the conductor including first Taylor ${ }_{490}$ cones. This leads to a second surge in CL and a significant decrease in PD. The observations with the UV scope (cf. Fig.7) support that in the first two minutes discharges primarily originate from the upper side of the conductor whereas later on they start almost exclusively from the 495 drops at the lower edge.

The duration of the transition from the phase in which water drops on the upper surface dominate the corona characteristics and the start of the formation of drops on the lower edge is likely to be strongly influenced by the rain intensity. Low rain intensities most likely prolong this phase and could lead to more problematic PD as well $1_{500}$ as RI and AN characteristics.

\subsection{Quasi Steady State}

After 15 to 20 minutes, the drop profile settles down and most of the recorded quantities are already close to $\mathrm{o}^{505}$ their steady state values (cf. Figs.8 and 9). However, ion current still shows a gradual increase after being exposed to rain for more than half an hour. This coincides with an increase of the mean area of pendant drops.

Due to the low rates of change, it is difficult to say ${ }^{510}$ if a steady state was reached after $1 \mathrm{~h}$, but considerable changes for longer exposure to rain at this point seem un-

460 likely. Still, the time it takes until steady state is reached is expected to depend largely on rain intensity.

\subsection{Dry-Off}

Stopping the rain results in an immediate reaction of electrical and optical quantities (cf. Figs.8 and 9). Similar to the rain start phase, CL and PD behave quite differently. The PD level constantly increases until the con- ${ }^{52}$ ductor is almost completely dry. Compared to the steady state, it increases by a factor of two and reaches its maximum only shortly before the corona activity of the conductor ceases completely. The total ion current on the contrary initially decreases very rapidly. This correlates most ${ }^{525}$ closely with a similarly steep decrease of Taylor cones and mean projected drop area of drops in the "under" region.
Interestingly, during this phase the total number of detected drops below the conductor increases slightly, meaning some larger drops split up into smaller ones. Starting from around $t=90 \mathrm{~min}$ the corona current then decreases steadily and mostly linearly. Towards the end of the drying process, at around $t=140 \mathrm{~min}$ the curve flattens quite significantly.

During the drying phase, CL are in general related very closely with the number of drops on the lower side of the conductor, $n_{\text {under }}$. The two quantities show an almost directly proportional correlation without any offset for the complete drying process (Fig. 11), this is not the case for $n_{\text {on }}$.

The illustrated quantities indicate that the drying process generally consists of different phases. The fast decrease in current at the start can be connected to a rapid decrease in the number of Taylor cones, which might be a result of the lacking water supply. Subsequently, $n_{\text {under }}$ and current decrease in a very similar manner. More than an hour after the rain stopped, no cones are detected anymore, which seems to result in a slow down of the drying process with noticeably smaller rate of fall of current and drop number. The return of CL to the pre-rain background level coincides precisely with the disappearance of detectable drops on the lower side of the conductor.

\section{Discussion of the Method}

The presented image acquisition and processing methods allow for the extraction of valuable information about the relationship between the physical behavior of water drops and DC corona characteristics of a typical overhead line conductor. The method very accurately detected the first appearance of drops on the conductor surface as well as the subsequent merging and movement of liquid towards the lower side of the conductor. Furthermore, the algorithm produced very reliable information about the number, size and shape of drops suspended from the lower side of the conductor, as verified by manually reviewing a large number of images.

A separate method, using a UV corona scope, showed that just after the start of a rain shower, discharges originate from the upper side of the conductor. During steady state conditions, on the other hand, they originate primarily from the lower side. The evaluation of the data has not been exhausted yet. A more precise time synchronization between the data collected through the UV scope and the instantaneous shape of water drops recorded without the UV scope would certainly be of interest for a more detailed investigation of the relation between drop form and discharge characteristics.

The drop detection method has some limitations with regard to the detection of drops on the surface (area "on"). The contrast of the drops with respect to their background is relatively small for small drops, especially on the upper side of the conductor. This comes from an inhomogeneous illumination. Selecting detection parameters required a 
trade-off between being able to detect small individual of larger wet areas. Adjusting the detection parameters as a function of the total amount of water collected on the surface can reduce this problem. However, this constitutes a dynamic change in the detection parameters. The impact of such dynamic parameter adjustments would lead585 to more uncertainties in the interpretation of results, especially when analyzing data from different experiments and conductor types. It was decided that these uncertainties outweigh the benefits of such an approach. An improved smaller drops on the upper side of the conductor could probably be achieved by an improved UV il- ${ }^{590}$ lumination with additional lamps to enhance the contrast in this area.

The presented data was collected with new conductors under no-load conditions. Factors such as conductor surface aging, pollution, wind and ohmic heating under load ${ }^{595}$ conditions may have an impact on the corona behavior, especially in the drying phase. Carefully designed outdoor experiments are necessary to better assess their influence. Most aspects of the presented methods could in principle be used for such investigations.

\section{Conclusion}

With regard to the presented methods, the following conclusions can be drawn:

- The image acquisition and processing methods are able to accurately detect the number, size and shape of water drops on and below the conductor, verified by manually checking a large number of frames.

- A measure of the sharpness of suspended water drops ${ }^{61}$ can be automatically derived, referred to in this publication as the drop angle $\alpha$.

- Evaluation of data from a UV scope allows the determination of the precise location of corona discharges and the overlay of this information with images of ${ }_{615}$ the wet conductor.

Evaluating the optical data obtained during and after a one hour rain period of $12 \mathrm{~mm} / \mathrm{h}$ for a stranded conductor under positive DC voltage, that was originally only ${ }^{620}$ intended to test the presented method, already yields the following conclusions:

- In the first few minutes after the rain starts, dis- ${ }^{625}$ charges originate primarily from small drops on the upper side of the conductor. The partial discharge amplitude during this phase significantly overshoots its steady state value.

- The subsequent appearance of drops on the lower side of the conductor reduces the PD amplitude but increases CL.
- A statistical evaluation of the cone angle $\alpha$ of pendant drops showed a characteristic minimum at around $99.5^{\circ}$, which is very close to the value for a balance of electrostatic forces and surface tension $\left(\alpha=98.6^{\circ}\right)$, identified by Taylor [16]. Drops with an angle below this value are unstable and presumably produce discharges while those with a higher angle do not. Time-synchronized analysis of UV images and regular images will be able to verify this hypothesis.

- After the end of the rain phase, corona currents decrease to zero over a period of approximately two hours. During this phase there is an almost directly proportional relationship between CL and the number of drops on the lower side of the conductor. PD discharge amplitudes on the hand increase steadily over this same period until they nearly instantaneously drop to the pre-rain level when corona activity seizes altogether.

- The inverse relationship between CL and PD charge amplitude observed during the initial rain start and post-rain phase is in line with previous observations by [17, which show that smaller drop sizes lead to a decrease in discharge repetition rate (causing lower CL) and an increase in of pulse amplitudes (causing higher $\mathrm{PD}$ ).

Concluding, the presented methods allow interesting insights into the behavior of rain drops on overhead lines and the relationship with corona phenomena. The targeted future application is to gain a deeper understanding of differences observed in DC corona characteristics of different conductor surface types [22] and to derive ideas for improved designs.

\section{References}

[1] ENTSO-E, 10-Year Network Development Plan, 2014.

[2] B. Rusek, C. Neumann, S. Steevens, U. Sundermann, K. Kleinekorte, J. Wulff, F. Jenau, K. H. Weck, Ohmic coupling between AC and DC circuits on hybrid overhead lines, in: Cigre Auckland Symposium, Auckland, 2013.

[3] T. Guillod, M. Pfeiffer, C. M. Franck, Improved Coupled IonFlow Field Calculation Method for AC/DC Hybrid Overhead Power Lines, IEEE Transactions on Power Delivery 29 (6) (2014) 2493-2501. doi:10.1109/TPWRD.2014.2322052

[4] B. A. Clairmont, G. B. Johnson, L. E. Zaffanella, The effect of HVAC-HVDC line separation in a hybrid corridor, Power Delivery, IEEE Transactions on 4 (2) (1989) 1338-1350. doi: 10.1109/61.25621

[5] M. Heindl, M. Beltle, S. Tenbohlen, U. Sundermann, F. Schatzl, Betriebsverhalten von Leistungstransformatoren in Hybridnetzen, in: VDE-Kongress 2012, VDE VERLAG GmbH, 2012.

[6] G. Johnson, Electric Fields and Ion Currents of a $\pm 400 \mathrm{kV}$ HVDC Test Line, IEEE Transactions on Power Apparatus and Systems PAS-102 (8) (1983) 2559-2568. doi:10.1109/TPAS. 1983.317775

[7] R. M. Morris, B. Rakoshdas, An Investigation of Corona Loss and Radio Interference from Transmission Line Conductors at High Direct Voltages, IEEE Transactions on Power Apparatus and Systems 83 (1) (1964) 5-16. doi:10.1109/TPAS.1964. 4765917 
[8] B. Bailey, Progress Report on BPA HV DC Test Line Radio Noise and Corona Loss, IEEE Transactions on Power Apparatus and Systems PAS-86 (10) (1967) 1141-1145. doi: 10.1109/TPAS.1967.291865

[9] P. Maruvada, N. Trinh, D. Dallaire, N. Rivest, Corona performance of a conductor bundle for bipolar HDVC transmission at $\pm 750 \mathrm{kV}$, IEEE Transactions on Power Apparatus and Systems 96 (6) (1977) 1872-1881. doi:10.1109/T-PAS.1977.32521

${ }_{645}[10]$ V. Chartier, Electrical environment of the uprated Pacific NW/SW HVDC Intertie, Power Delivery, IEEE Transactions on 4 (2) (1989) 1305-1317.

[11] F. Hirsch, E. Schafer, Progress Report on the HVDC Test Line of the $400 \mathrm{kV}$-Forschungsgemeinschaft: Corona Losses and Ra-

650 dio Interference, IEEE Transactions on Power Apparatus and

(1) Systems PAS-88 (7) (1969) 1061-1069. doi:10.1109/TPAS. 1969.292506

[12] N. Hylten-Cavallius, S. Annestrand, H. Witt, V. Madzarevic, Insulation Requirements, Corona Losses, and Corona Radio Interference for High-Voltage D-C Lines, IEEE Transactions on Power Apparatus and Systems 83 (5) (1964) 500-508. doi:10.1109/TPAS.1964.4766030

[13] J. Zeleny, The electrical discharge from liquid points, and a hydrostatic method of measuring the electric intensity at their surfaces, Physical Review III (1914) 1-1.

[14] W. Macky, Some investigations on the deformation and breaking of water drops in strong electric fields, Proceedings of the Royal Society of London 133 (822) (1931) 565-587.

[15] W. English, Corona from a water drop, Physical Review 74 (2) (1948) 1-1. doi:dx.doi.org/10.1103/PhysRev.74.179

[16] G. Taylor, Disintegration of Water Drops in an Electric Field, Proceedings of the Royal Society A: Mathematical, Physical and

Engineering Sciences 280 (1382) (1964) 383-397. doi:10.1098/ rspa.1964.0151

670 [17] M. Akazaki, Corona Phenomena from Water Drops on Smooth Conductors under High Direct Voltage, IEEE Transactions on Power Apparatus and Systems 84 (1) (1965) 1-8. doi:10.1109/ TPAS.1965.4766101

[18] G. Dawson, Electrical corona from water drop surfaces, Journal of Geophysical Research 75 (1970) 1-1.

[19] J. Hoburg, J. Melcher, Current-driven, corona-terminated water jets as sources of charged droplets and audible noise, IEEE Transactions on Power Apparatus and Systems 94 (1) (1975) 128-136. doi:10.1109/T-PAS.1975.31835.

${ }_{680}[20]$ M. Hara, S. Ishibe, M. Akazaki, Corona discharge and electrical charge on water drops dripping from DC transmission conductors - an experimental study in laboratory, Journal of Electrostatics 6 (1979) $235-257$.

[21] M. Hara, M. Akazaki, Onset mechanism and development of corona discharge on water drops dripping from a conductor under high direct voltage, Journal of Electrostatics 9 (1981) 339353.

[22] M. Pfeiffer, C. Franck, Impact of conductor surface type and rain intensity on hvdc corona losses, Power Delivery, IEEE Transactions on PP (99) (2015) 1-1. doi:10.1109/TPWRD.2015. 2424315

[23] T. Schultz, M. Pfeiffer, C. M. Franck, Ion flow simulations of HVDC conductors under rainy conditions, in: IET Conference on AC and DC Power Transmission, Birmingham, 2015.

695 [24] U. Straumann, Mechanism of the tonal emission from ac high voltage overhead transmission lines, Journal of Physics D: Applied Physics 44 (7) (2011) 075501. doi:10.1088/0022-3727/ 44/7/075501

[25] T. P. Regmi, A. L. Thompson, Rainfall Simulator for Laboratory Studies, Applied Engineering in Agriculture 16 (6) (2000) 641-647.

[26] Forsyth Electro-Optics, Inc., The corona scope - instruction manual (1999). 